\title{
I 43 - THE USE OF POROUS MEDIUMS CONNECTIVITY CONCEPT FOR TRABECULAR BONE QUALITY EVALUATION
}

ACA Souza, MD, PhD, Institute of Geriatrics, Pontificial Catholic Univ; D Barbieri, Institute of Geriatrics, Pontificial Catholic University RS; R Schneider, Institute of Geriatrics, Pontificial Catholic University RS; Waldir L. Roque, Instituto de Matemática UFRGS; F Rodrigues Introduction:The mechanical competence of bone is a function bone mass and architecture but BMD alone is not able to reflect bone quality. The modeling of porous mediums, is an important method for the comprehension of the physical and geometrical properties of trabecular bone. Objectives: Develop a technique to identify the connectivity of the trabecular three-dimensional porous space by using the study of dissectors. Methods:The connectivity between porous of neighbor slices is identified in the orthogonal direction. The three-dimensional porous space connectivity is calculated by using the Euler-Poincaré volumetric characteristics technique. Seven cadaver collected vertebrae were scanned by Hologic 4500A DXA and by a Siemens Somatom Plus CT by using a resolution of $512 \times 512$ pixels, slices of $2.0 \mathrm{~mm}$ thickness, setting of $120 \mathrm{kV}$ and $110 \mathrm{~mA}$. The images were segmented by using image processor software and the Euler-Poincaré derived index was calculated for every vertebra.

Vertebrae I and 2 showed low density $(T-s c o r e<2,5)$ and the Euler-Poincaré derived index confirmed low connectivity, revealing architectural deterioration. Vertebrae 3 and 4 had an osteopenic BMD values, but good trabecular connectivity. Vertebrae 5, 6 and 7 had T-score $>$ $I$ and good Euler-Poincaré derived index. Despite vertebra 5 had lower BMD than 6 and 7, its trabecular connectivity index was better than 6 and 7 .

These results can demonstrate that study of trabecular porous connectivity provides important information for clinical decision related to bone structural quality. 